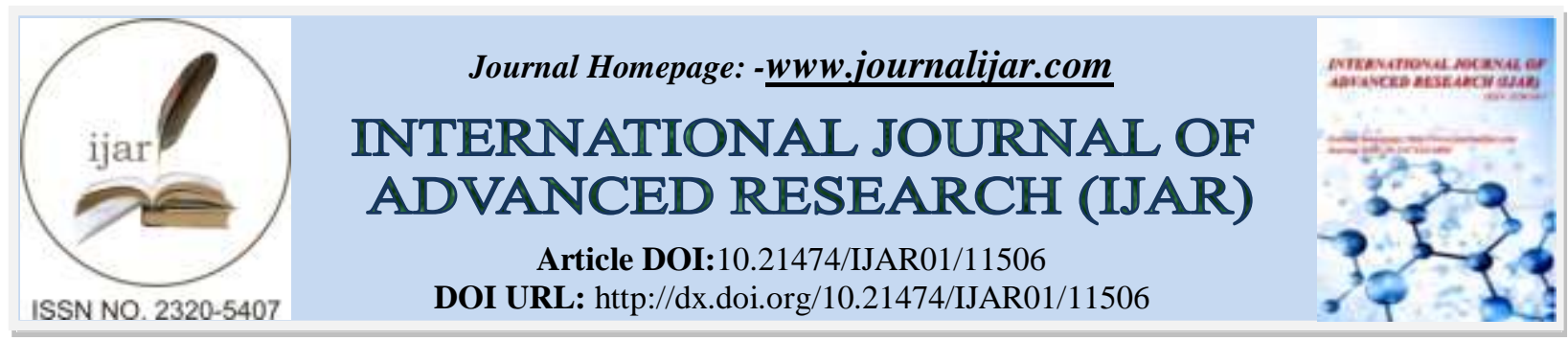

RESEARCH ARTICLE

\title{
EVALUATION OF DOSIMETRIC PARAMETERS FOR ADOLESCENT CRANIOSPINAL IRRADIATION
}

\author{
Rajni Verma ${ }^{1}$, Gourav Kumar Jain ${ }^{2}$ and Arun Chougule ${ }^{1}$ \\ 1. Department of Radiological Physics, SMS Medical College \& Hospital, Jaipur-302004 India. \\ 2. Department of Radiation Oncology, Shalby Multi Speciality Hospital, Jaipur-302021, India.
}

\section{Manuscript Info}

..........................

Manuscript History

Received: 10 June 2020

Final Accepted: 14 July 2020

Published: August 2020

Key words:-

Craniospinal Irradtion, RapidArc,

Dosimetry

\section{Abstract}

Background: Craniospinal irradiation (CSI) is an important and challenging radiotherapy technique used for the treatment of Medulloblastoma, one of the most common pediatric cancers. Pediatric patients present with wide variability in weight, height, spine length, brain diameter, body size, intra and inter fractional motion depending on their age as compared to adults. These parameters have significant impact on radiation dose delivery to target and normal structures during radiotherapy of pediatric cancer patients. The present study was aimed to evaluate dosimetric parameters and generate plan quality indices for adolescent CSI.

Materials and Methods: A 17 years old male adolescent patient with Medulloblastoma was recruited in the present sturdy. The patient was planned with RapidArc ${ }^{\mathrm{TM}}$ / Volumetric Modulated Arc Therapy (VMAT) mode using 6 MV X-Ray photon beam from Varian Trilogy with FFF machine. The dose points such as Dmax, Dmean, $V 2 \%, V 98 \%$, $V 95 \%, V 105 \%$ and $V 107 \%$ were collected using dose statistics and dose volume histogram (DVH) and analyzed for calculation of various dosimetric parameters. The in-vivo dosimetry was performed and radiation doses to eyes were assessed using CaSO4:Dy TLD powder.

Results: The plans were approved with a gamma passing rate $(3 \%$, $3 \mathrm{~mm}$ ) of more than $95 \%$. The dose homogeneity index and conformity index were observed 0.074 and 1.10 respectively. The average doses to eyes were observed approximately $35 \%$ of the tumor dose during RapidArc $^{\mathrm{TM}}$ - CSI. The results of the present study demonstrated that V5 was observed high the normal structures except kidneys. Esophagus and thyroid were receiving significant low dose spillage at a cost of high homogeneity and conformity of dose to PTV which were needed to enhance tumor control probability (TCP) of Medulloblastoma.

Conclusion: The present study developed a benchmark guide for adolescent RapidArc ${ }^{\mathrm{TM}}$ - CSI.

Copy Right, IJAR, 2020,. All rights reserved.

\section{Introduction:-}

Non-communicable diseases (NCDs) accounts for $71 \%$ of total deaths worldwide [1]. In India, NCDs are estimated to account for $63 \%$ of all deaths. Cancer is second only to cardiovascular diseases (CVDs) as one of the leading 
causes amounting to $9 \%$ [2]. Central nervous system (CNS) neoplasms are second most common pediatric cancers as hematologic malignancies are most common pediatric cancers. However, CNS malignancies constitute the single largest source of disability and death from cancer in children and young adults worldwide [3]. Craniospinal irradiation (CSI) is an important method of radiotherapy (RT) for the treatment of some types of central nervous system (CNS) malignancies in young children for example medulloblastoma and germ cell tumors. CSI is a challenging RT technique and require a great care in the radiotherapy planning, delivery and radiation field junction dose verification for optimal results. [4-7]. In-vivo dosimetry is the recommended procedure to assess and verification of radiation doses during radiotherapy [8-9]. The present study was aimed to perform in-vivo dosimetry during CSI to assess radiation dose for organs at risk (OAR). The present study planned to evaluate various dosimetric parameters and generate plan quality indices for adolescent CSI.

\section{Materials and Methods:-}

\section{Research study design:}

Prospective, Cross sectional, single centre research study design was used to assess dosimetric parameters of RapidArc ${ }^{\mathrm{TM}}-$ CSI.

\section{Criteria for selecting the sample: \\ Inclusion Criteria:}

Adolescence CSI patients who were willing to participate in the study and within the age group of 12-18 years after obtaining the informed consent.

\section{Exclusion Criteria:}

CSI patients who belong to age less than 12 years and more than 18 years.

\section{Patient characteristics and CT simulation:}

A 17 years old male patient with post surgery status of Medulloblastoma diagnosis was included in the study. CT scan of the patient from the skull vertex to coccyx in supine position using immobilization device on Somatom Scope 32 slice multislice CT scanner (Siemens Shenghai Medical Equipment Limited, China) was done with 3-mm slice thickness. CT images were analyzed for contouring on SomaVision workstation (Varian Medical Systems, Inc., Palo Alto, CA, USA) where the target volumes (PTV Brain, PTV Spine) and normal structures were delineated by radiation oncologist as per the recommended guidelines of ICRU62 and Children's Oncology Group [10-12].

\section{Treatment planning and radiation dose prescription:}

The patient treatment plan was generated on Eclipse treatment planning system (TPS) version 13.7 (Varian Medical Systems, Inc., Palo Alto, USA) with RapidArc ${ }^{\mathrm{TM}}$ mode $6 \mathrm{MV}$ X-Ray photon energy and dual arc $\left(2\right.$ full arc, $179^{\circ}-$ $181^{\circ}, \pm 30^{\circ}$ collimator angle) technique for a maximum dose rate of $600 \mathrm{MU} / \mathrm{Minutes}$ using Trilogy (Varian Medical Systems, Inc., Palo Alto, USA) linear accelerator (Linac) equipped with 120 Millennium Multi-Leaf Collimator (MLC) as shown in Figure 1. The two Arcs were delivered in opposite rotations (clockwise and counterclockwise). Collimator was set to rotate to a value other than zero in order to avoid tongue and groove effect. The patient was planned to treat with external beam radiotherapy Rapid Arc ${ }^{\mathrm{TM}}$ mode with a conventional fractionation regime with radiation dose prescription of $36 \mathrm{~Gy}$ in 20 fractions to planning target volume (PTV), with a dose delivery of 1.8 Gy/fraction. A maximum field size of $40 \times 40 \mathrm{~cm}^{2}$ can be possible with the 120 Millennium MLC. The PTV spine length was $67.9 \mathrm{~cm}$ and the total target volume was recorded $2767 \mathrm{~cm}^{3}$ in the present study. When spine length is greater than $40 \mathrm{~cm}$, the treatment planning demands to place three isocenter due to mechanical limitations of treatment machine and therefore three treatment plans were generated. Volumetric modulated arc therapy (VMAT) plan optimization was performed using Photon Optimizer (PO version 13.7.16). Volume dose calculation was performed using the analytical anisotropic algorithm (AAA, version 13.7.16), a Model based superpositionconvolution algorithm implemented using the Eclipse software. The calculation of dose grid was based on a grid resolution of $2.5 \mathrm{~mm}$.

\section{Treatment plan quality indices:}

The patient specific quality assurance (QA) was performed using Varian amorphous silicon (aSi) portal dosimetry with standardized portal dose image prediction (PDIP) algorithm configuration. The dose points such as maximal dose point (Dmax), mean dose (Dmean), V2\%, V98\%, V95\%, V105\% and V107\% were collected using dose statistics and dose volume histogram (DVH) and analyzed for calculation of various dosimetric parameters. The dose indices of approved plan were evaluated using PTV conformity index (CI) and dose homogeneity index (HI), 
normal tissue (NT) dose statistics, integral dose (ID) as per ICRU83 [13]. The summary of radiotherapy treatment plan for patient was presented in Table 1 .

\section{In-vivo dosimetry:}

The thermoluminesence (TL) dosimeter system used in the study was a commercial TL reader system with $\mathrm{CaSO}_{4}$ : Dy powder, manufactured by Nucleonix Ltd., India and is shown in Figure 2. The calibration for TLD powder was carried out in Cobalt-60 reference beam with traceability to the primary standard. The volume of TLD powder was $70.7 \mathrm{~mm}^{3}$ used in the present study. The dosimeters were irradiated in groups of 3 pairs to known dose values. The energy independence with reference to $6 \mathrm{MV}$ X-ray beam used in the clinical setting of RT was checked and the linear dose response was established. The powder form of dosimeter is used in the present study because of its high sensitivity and it is a good choice of detector for scatter and leakage dose measurements at a cost of optimal accuracy in the measurement due to uncertainty associated with mass/volume measurements. However, a great precaution is taken during TL powder measurements and steel rods of identical volume provided by manufacturer were used in the present study for accurate powder sample measurement and transfer mechanism.

Thermoluminescence dosimeters were used to measure the radiation doses during RapidArc ${ }^{\mathrm{TM}}-\mathrm{CSI}$ treatment. The eye doses were assessed by placing the dosimeter as close as possible to the eye at eyebrow level. The measurements for each patient have been performed for 3 consecutive RT treatment fractions and average reading was considered as eye dose per fraction in order to reduce uncertainty in measurement.

\section{Results and Discussion:-}

All the treatment plans were approved with an area gamma passing rate greater than $95 \%(99.4 \%$ for Spine lower plan, $99.3 \%$ for Spine upper plan, $99.9 \%$ for Brain plan) for gamma analysis dose tolerance $3 \%$ and distance to point agreement (DTA) $3 \mathrm{~mm}$ criteria. The PTV coverage was achieved with $95 \%$ of prescribed dose and PTV volume received $98 \%$ of prescribed dose received (D98\%) was recorded $97 \%$ of PTV. The typical distribution of radiation dose was presented in Figure 3. The dose homogeneity index and conformity index were observed 0.074 and 1.10 respectively. The total body integral dose was $390 \mathrm{~J}$ when a constant density $1 \mathrm{~g} / \mathrm{cm}^{3}$ was assumed. The TPS calculated mean dose to right eye and left eye were recorded $13.45 \mathrm{~Gy}$ and $15.00 \mathrm{~Gy}$ respectively during the whole treatment fractions. Whereas, the right eye and left eye were received TLD estimated total dose of 11.82 Gy and 13.34 Gy respectively. The TPS mean doses were found approximately $12 \%$ higher than doses measured with TLD. The possible cause of this under response of TLD measurement is that the TLDs were placed at a distance from tumor near to the side of the eyes. The average doses to eyes were observed approximately $35 \%$ of the tumor dose during RapidArc ${ }^{\mathrm{TM}}$ - CSI. The dosimetric parameters for OAR with RapidArc ${ }^{\mathrm{TM}}$ - CSI were evaluated and presented in Table 2.

\begin{tabular}{|c|c|c|c|}
\hline Organ & V5 & V10 & V20 \\
\hline Heart & 99 & 23 & 0 \\
\hline Rt lung & 98 & 31 & 4 \\
\hline Lt lung & 98 & 30 & 3 \\
\hline Rt kidney & 75 & 21 & 1 \\
\hline Lt kidney & 70 & 17 & 1 \\
\hline Liver & 83 & 100 & 0.5 \\
\hline Esophagus & 100 & 100 & 90 \\
\hline Thyroid & 100 & & 13 \\
\hline
\end{tabular}

The results of the present study demonstrated that V5 was observed high in most of the OARs except kidneys. Esophagus and thyroid were receiving significant low dose spillage at a cost of high homogeneity and conformity of dose to PTV which were needed to enhance tumor control probability (TCP) in case of Medulloblastoma RT treatment. 


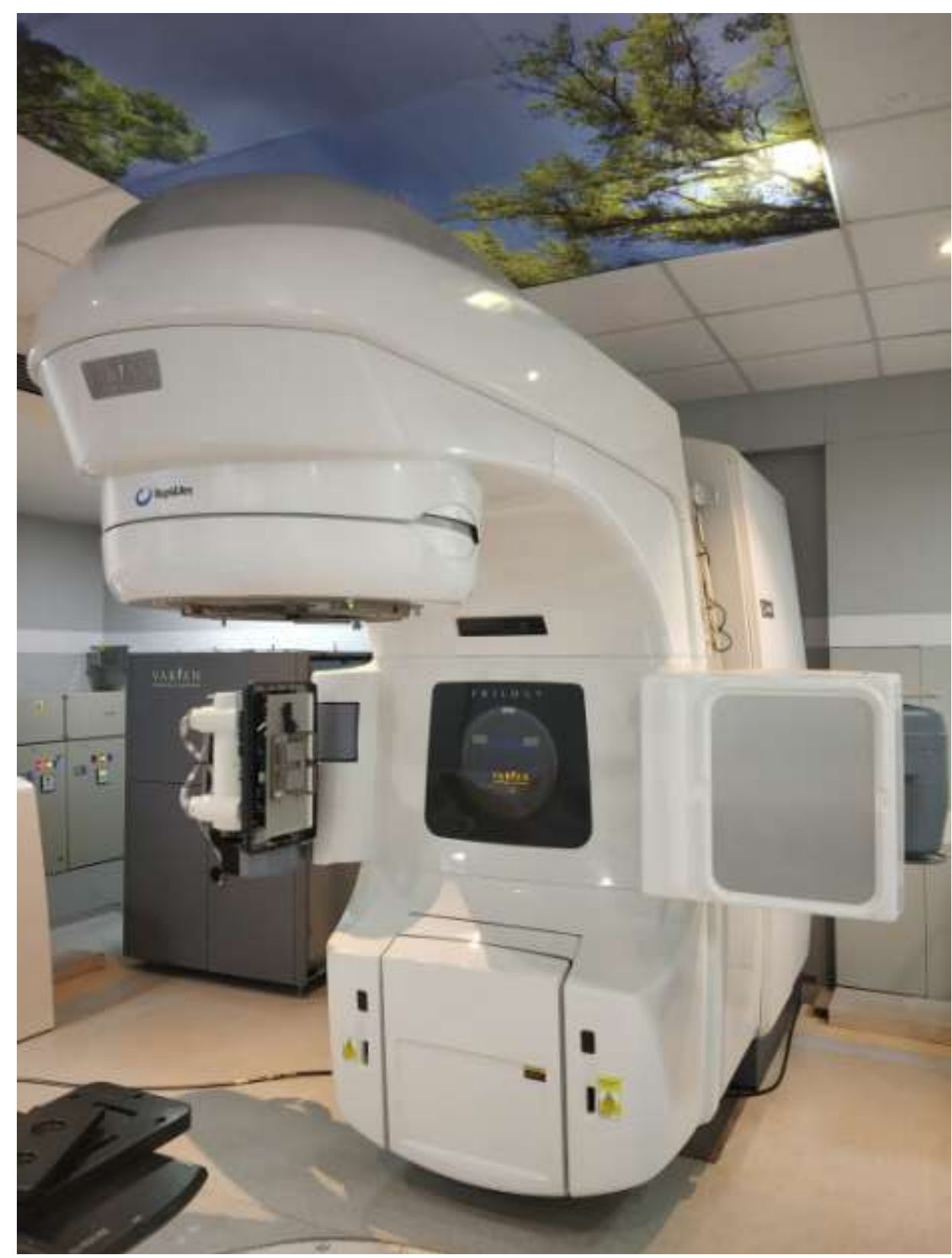

Figure 1:- Varian Trilogy with FFF unit with Millennium 120 MLC equipped with image guided radiotherapy (IGRT) modalities cone beam computed tomography (CBCT) and Megavoltage imaging with trade name On-Board Imaging (OBI) and Electronic Portal Imaging Device (EPID) respectively. Planar kV and CBCT imaging with OBI was used for IGRT in the present study. 
Table 1:- A brief description of radiotherapy treatment plan used in the present study.
Patient
characteristics
17 year old patient with post surgery status of
CT scan $3 \mathrm{~mm}$ slice thickness in supine position using immobilization device
Contouring
Target structures and OARs contoured as per recommended guidelines of ICRU62 and Children's Oncology Group.
Treatment unit
Varian Trilogy with FFF equipped with 120 Millennium MLC
Treatment
Planning
Dose prescription
Treatment Delivery

\section{Patient specific QA}
Eclipse version 13.7 with AAA dose calculation algorithm and dose calculation grid $2.5 \mathrm{~mm}$

$$
36 \text { Gyl } 20 \text { Fractions }
$$
RapidArc mode with dual arc delivery and three isocenter technique
Varian amorphous silicon (aSi) portal dosimetry with portal dose image prediction (PDIP) algorithm configuration

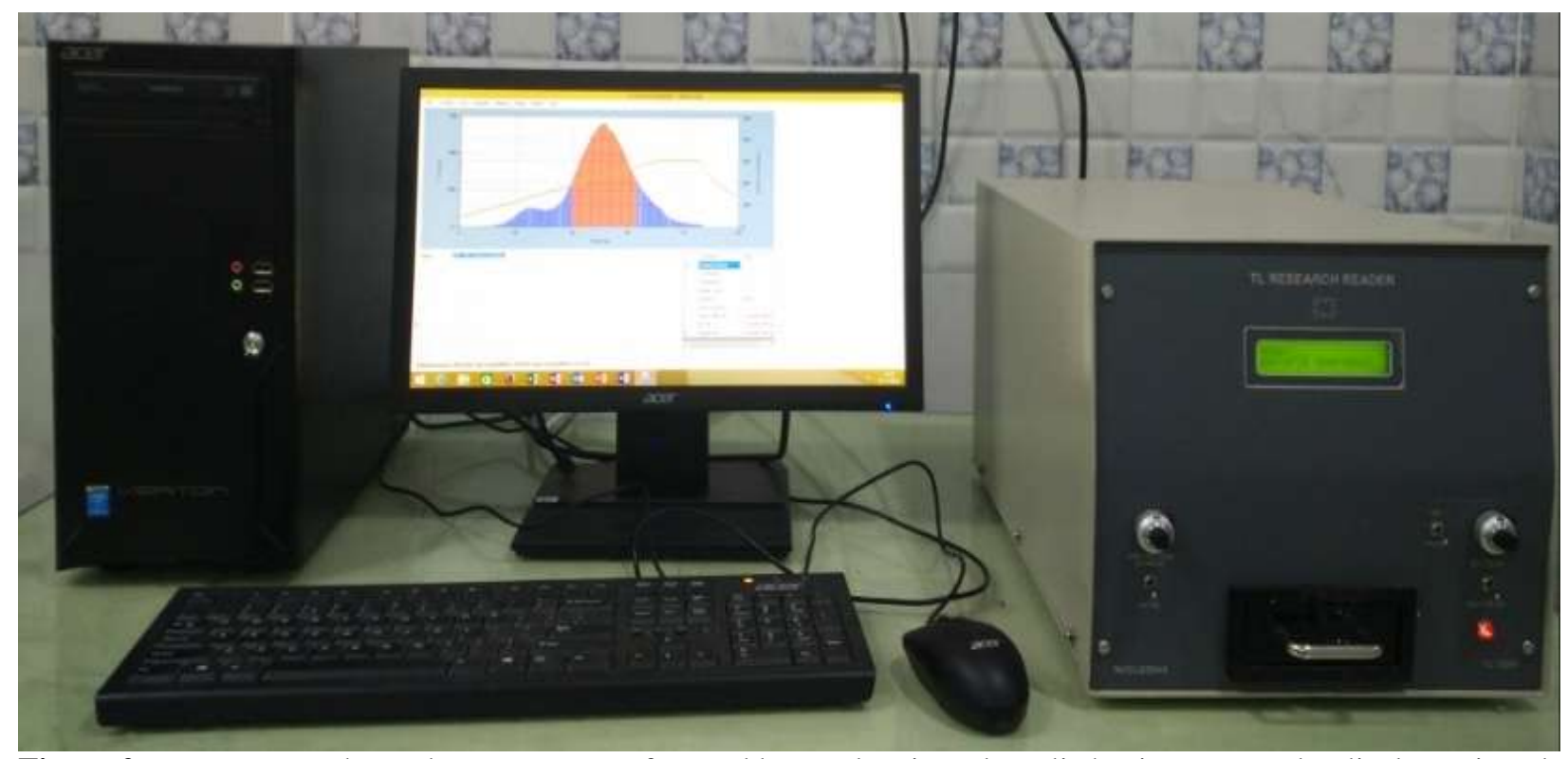

Figure 2:- TLD Research Reader system manufactured by Nucleonix Ltd., India having TL Reader display unit and computer system. Measurements were obtained with $728 \mathrm{~V}$ photo multiplier tube (PMT) applied voltage. Holders of different depths were used for accurate estimation of amount of CaSO4:Dy powder and $70.7 \mathrm{~mm}^{3} \mathrm{TLD}^{\mathrm{T}}$ powder was used in the study. 


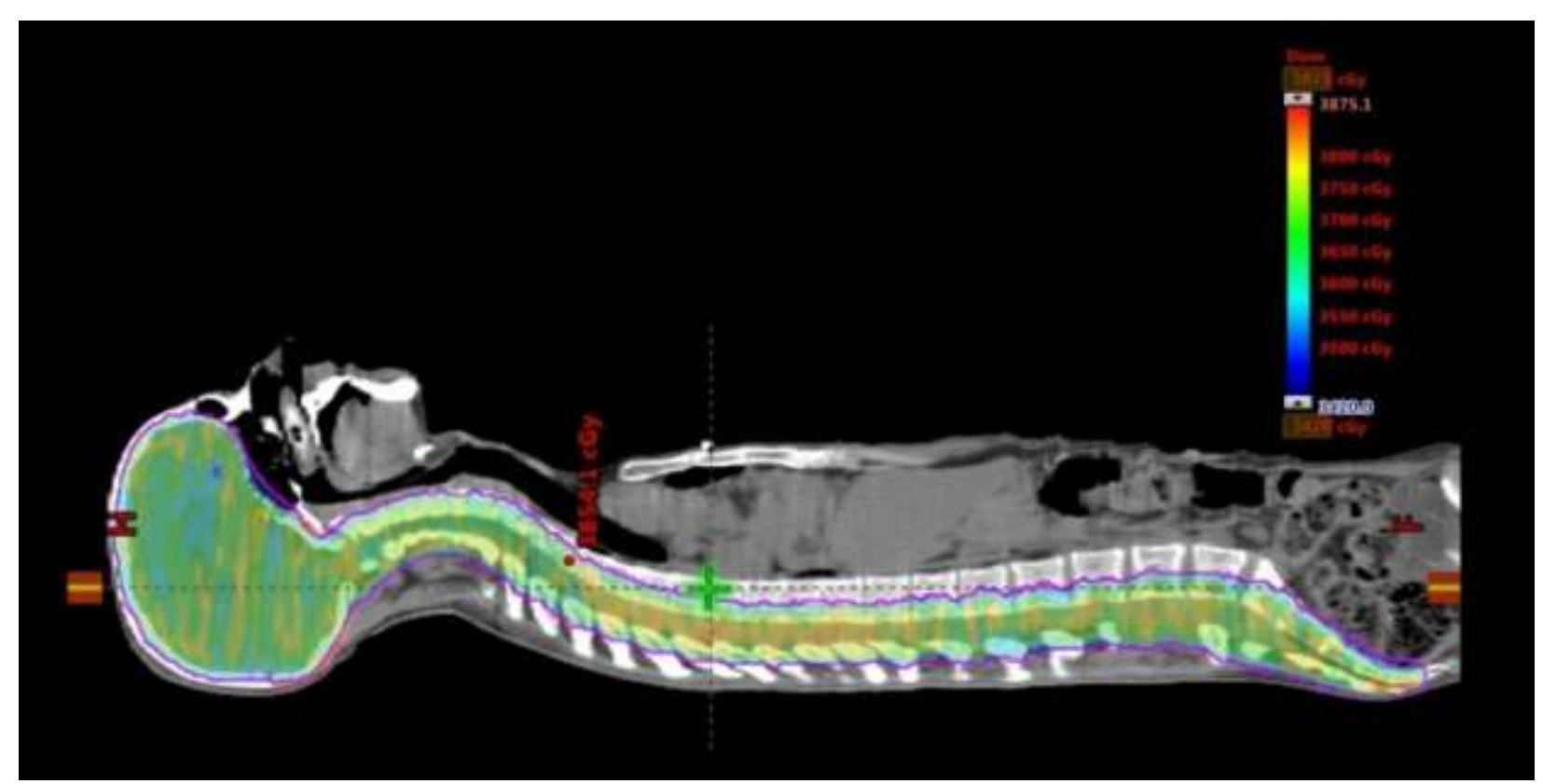

Figure 3:- Radiation dose distribution with 95\% of prescribed radiation dose to PTV in Sagittal view of CT scan slice with spine length $67.9 \mathrm{~cm}$.

\section{Conclusion:-}

The present study provided results to evaluate RapidArc ${ }^{\mathrm{TM}}$ - CSI plans for an adolescent patient. RapidArc ${ }^{\mathrm{TM}}$ is a valid treatment option for CSI treatment with ease of treatment delivery despite of increased planning time. This study showed from the dosimetric point of view the feasibility and technical appropriateness of RadpidArc ${ }^{\mathrm{TM}}$ - CSI for the treatment of Medulloblastoma. The present study developed a benchmark guide for adolescent $\operatorname{RapidArc}^{\mathrm{TM}}$ CSI.

\section{Acknowledgment:-}

The authors acknowledge administration and staff of Shalby Multi-Specialty Hospital, Jaipur for providing support and cooperation to perform the study.

\section{Conflict of interest:}

Nil

\section{References:-}

1. WHO: World Health Statistics 2019: Monitoring Health for the SDGs. Geneva, Switzerland, World Health Organization, 2018 (Downloaded from : https://www.who.int/gho/publications/world_health_statistics/2019/en/ Assess date: 14 August 2020)

2. Mathur P, Sathishkumar K, Chaturvedi M, Das P, Sudarshan KL, Santhappan S et al. Cancer

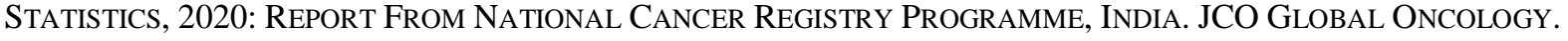
2020;6:1063-1075. DOI: 10.1200/GO.20.00122

3. Ward E, DeSantis C, Robbins A, Kohler B, Jemal A. Childhood and adolescent cancer statistics, 2014. CA Cancer J Clin. 2014;64(2):83-103.

4. Sharma DS, Gupta T, Jalali R, Master Z, Phurailatpam RD, Sarin R. High-precision radiotherapy for craniospinal irradiation: evaluation of three-dimensional conformal radiotherapy, intensity-modulated radiation therapy and helical Tomotherapy. Br J Radiol. 2009;82(984):1000-1009. doi: 10.1259/bjr/13776022

5. Pichandi A, Ganesh KM, Jerrin A, Balaji K, Surega A. Use of Flattening Filter-Free Photon Beams in Treating Medulloblastoma: A Dosimetric Evaluation. ISRN Oncology. 2014:1-5. (Article ID: 769698) doi: http://dx.doi.org/10.1155/2014/769698

6. Saleh H, Ferjani S, Masssey V, Howlin T, Pokhrel D. In Vivo Dosimetry and Daily Arc Junction Verification Using EBT3 Gafchromic Film for Pediatric Cranio-Spinal Irradiation. Med Phys. 2015;42:3525-3525. doi:10.1118/1.4925179 
7. Kim JY, Park SK, Kim YL, Suh TS, Shin JW, Oh KM, et al. Feasibility study of an optically-stimulated luminescent nanodot dosimeter (OSLnD) in high-energy photon beams. Journal of the Korean Physical Society. 2014;65:1159-1163. doi: https://doi.org/10.3938/jkps.65.1159

8. Yorke E, Alecu R, Ding L, Fontenla D, Kalend A, Kaurin D, et al. Diode in vivo dosimetry for patients receiving external beam radiation therapy. Report of Task Group No. 62 of the Radiation Therapy Committee of the American Association of Physicists in Medicine. AAPM Report No. 87. Available from: https://www.aapm.org/pubs/reports/RPT_87.pdf. Accessed on 07th August 2020.

9. International Atomic Energy Agency. Development of procedures for in vivo dosimetry in radiotherapy. IAEA Human Health Reports No. 8. Vienna: IAEA, 2013. Available from: http://wwwpub.iaea.org/MTCD/Publications/PDF/Pub1606_web.pdf. Accessed on 07th August 2020.

10. International Commission on Radiation Units and Measurements (ICRU), "Prescribing, recording and reporting photon beam therapy," ICRU Report 62, International Commission on Radiation Units and Measurements (ICRU), Bethesda, Md, USA, 1999, (Supplement to ICRU Report 50): ICRU Publications.

11. Breneman JC, Donaldson SS, Constine L, Merchant T, Marcus K, Paulino AC, et al. The Children's Oncology Group Radiation Oncology Discipline: 15 Years of Contributions to the Treatment of Childhood Cancer. Int J Radiat Oncol Biol Phys. 2018;101(4):860-874. doi: 10.1016/j.ijrobp.2018.03.002. PMID: 29976498

12. Gupta T, Sarkar C, Rajshekhar V, Chatterjee S, Shirsat N, Muzumdar D, Pungavkar S, Chinnaswamy G, Jalali R. Indian Society of Neuro-Oncology consensus guidelines for the contemporary management of medulloblastoma. Neurol India. 2017;65(2):315-32. Available from: http://www.neurologyindia.com/text.asp?2017/65/2/315/20184 Accessed on: 07thAugust 2020.

13. International Commission on Radiation Units and Measurements (ICRU), "Prescribing, recording and reporting Intensity-Modulated photon beam therapy (IMRT)," ICRU Report 83, International Commission on Radiation Units and Measurements (ICRU), Bethesda, Md, USA, 2012: ICRU Publications. 